\title{
Aggressive Vertebral Haemangioma Leading to Paraparesis in A Pregnant Lady---A Case Report
}

\author{
Author \\ Dr Dhrubajyoti B P Gohain \\ Assistant Professor, Department of Radiodiagnosis \\ Assam Medical College \& Hospital, Dibrugarh, Assam 786002 \\ Email_drd_gohain@yahoo.com, Mobile number_9435033461
}

\begin{abstract}
Vertebral haemangioma (VHs) are benign tumours with rich vasculature. They are called aggressive when it is characterised by bone expansion, extra osseous extension of the tumour, disturbance of local blood flow and, in rare cases, compression fractures ${ }^{[1,2]}$. We report a 19-year-old pregnant woman, presented with back pain \& progressive weakness of the lower limbs during her pregnancy period. Magnetic resonance imaging (MRI) revealed a haemangioma in the vertebral body extending into the posterior appendage with soft tissue component extending into epidural space causing compression of the spinal cord leading to myelopathic changes (VH type IV, according to Tomita's surgical classification.)
\end{abstract}

Keywords: haemangioma, MRI, posterior appendages, myelopathy

\section{Introduction}

Haemangioma is the most common primary benign hamartoma. Vertebral haemangioma (VHs) are benign tumours with rich vasculature. They are the most common Tumours of the spine with an estimated incidence of $10 \%-12 \%$ of the population. Despite its high incidence, they are often asymptomatic and only around $1 \%$ are symptomatic. The majority of vertebral haemangioma occur in thoracic spine, whereas lumbar and cervical spines are also involved. Pregnancy is a well-recognized state during which asymptomatic vertebral haemangioma become symptomatic due to expansion.

Here we describe an uncommon case of aggressive vertebral haemangioma in the thoracic region during pregnancy of 26 weeks gestation causing compressive myelopathy secondary to epidural extension.

\section{Case Report}

A 19 year pregnant lady was referred to the department of the radio-diagnosis for MRI spine. The chief complaint of the patient was gradual onset of paraparasis along with numbness \& tingling sensation in the lower limbs during her pregnancy period. She had no fever, vomiting, weight loss or any other significant findings.

MRI of the whole spine revealed a lesion in the D10 vertebral body involving the posterior appendage. The lesion was predominantly hypointense on T1 weighted images. Some T1 
hyperintense foci are noted within the hypointense vertebrae suggest fat component because they are suppressed on STIR sequences. T2 fat suppressed weighted images reveals hyperintensity involving whole vertebral body as well as the posterior appendages suggestive of predominant water content of the lesion. There is a T2 hyperintense soft tissue component in the epidural space behind the vertebral body causing compression of the spinal cord leading to myelopathic changes in the cord as evidenced by $\mathrm{T} 2$ hyperintensity in the cord at this level. Cortical breach of the vertebral body is noted posteriorly at this place. Some signal void linear areas are noted within the lesion in vertebra suggestive of thickened trabeculae. Metastasis of the spine was considered but excluded because she was too young, there were no constitutional symptoms and the presence of hyperintense signals on T1 weighted image favors benign etiology. Also, the vertebral height was preserved, again suggesting benign pathology. Metastasis of the spine usually shows destruction of the trabeculae

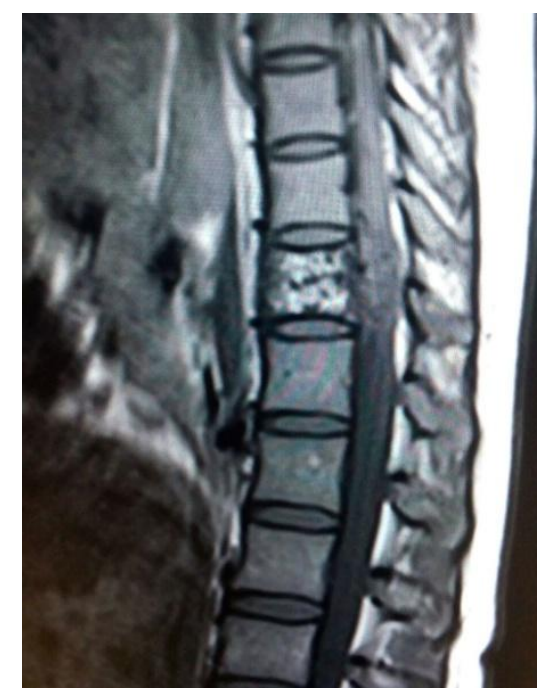

Fig:1

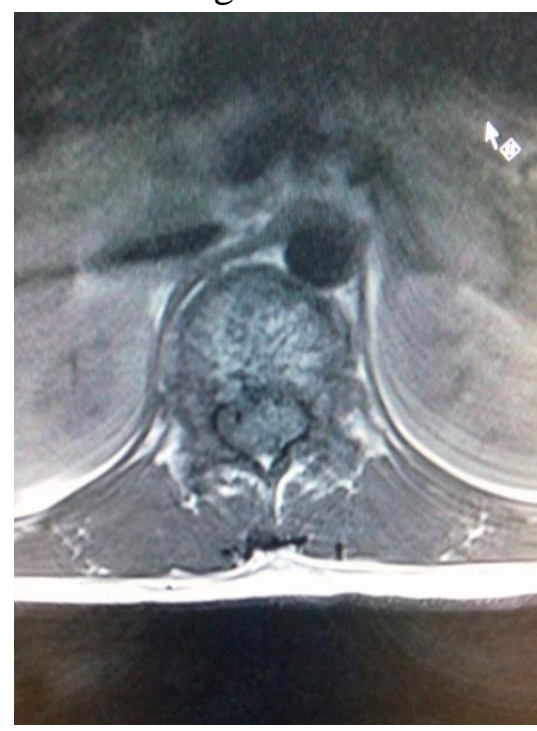

Fig:3

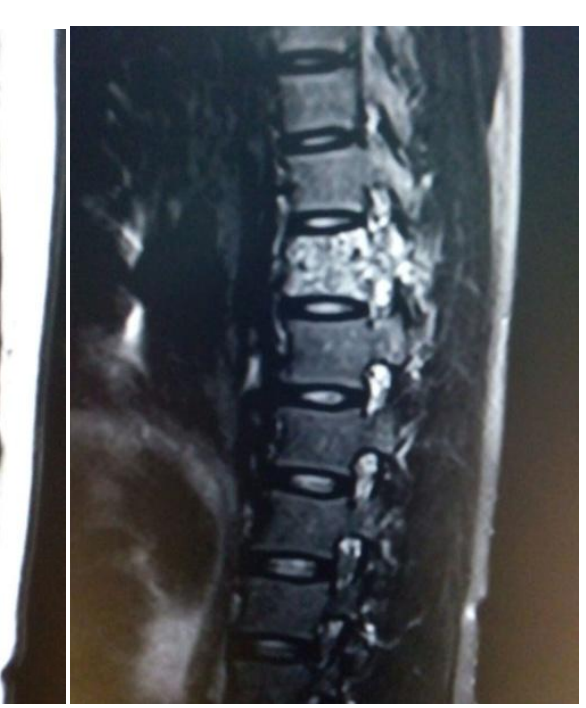

Fig:2

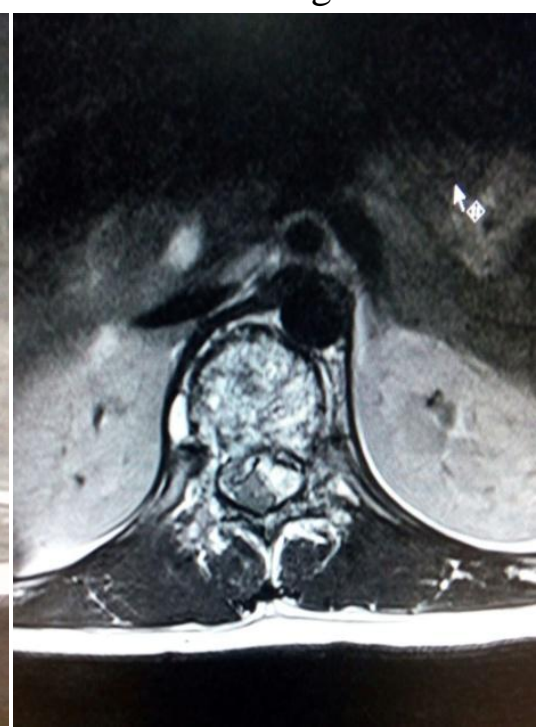

Fig:4

Fig 1,3---- T1 weighted images in segittal \& axial section

Fig 2,4---- T2 weighted images in segittal \& axial section. 


\section{Discussion}

Most of symptomatic vertebral hemangioma usually present during the third trimester and may show improvement in the postpartum period. The physiological factors including vascular, hormonal and altered hemodynamic act to enlarge the preexisting vertebral haemangioma ${ }^{[6]}$. Venous obstruction and increased intra-abdominal pressure caused by gravid uterus cause redistribution and increased blood flow through vertebral venous plexus, leading to growth and expansion of pre-existing vertebral hemangiomas [6]. In vertebral haemangioma, cord compression may be produced by one or more of the following mechanisms: (i) narrowing of spinal canal by the expansion of tumor within the vertebral body and/or posterior elements, (ii) subperiosteal growth of haemangioma forming an epidural mass, (iii) acute hemorrhage into the epidural space and (iv) rarely, compression fracture of the involved vertebral body. Compression fracture in haemangioma is unusual because the involved vertebra usually have thickened vertical trabeculae, which can withstand axial collapse. Vertebral haemangioma typically shows a coarse trabecular pattern, described as a 'corduroy' appearance on plain radiographic films. On axial computed tomography, this coarse trabecular pattern is characteristically seen as dense spots within a hypodense lesion, referred to as a 'polka dot' pattern. On MRI, benign incidental haemangioma usually appear as hyperintense on both T1- and T2-weighted images due to high fat component and less vascular stroma. On the other hand, aggressive haemangioma are usually hypointense to isointense on T1-weighted images and hyperintense on $\mathrm{T}_{2}$-weighted images due to prominent hypervascular stroma and less amount of fat ${ }^{[1,3,4]}$. MRI is also helpful in showing paravertebral and epidural extension of the lesion. Extraosseous components lack fatty tissue and thus appear isointense on T1 -weighted images.

There are three categories and seven types of VHs, according to Tomita's surgical classification of spinal tumours. In the first category, the hemangioma is limited to vertebral body (type I), with pedicle extension (type II), body-lamina extension (type III). In the second category, $\mathrm{VH}$ is extra-compartmental: spinal canal extension (type IV), paravertebral (type V), and adjacent vertebral extension (type VI). In the third category, the VHs are multiple.

\section{References}

1. Chen, H.I., Heuer, G.G., Zaghloul, K., Simon, S.L., Weigele, J.B. and Grady, M.S. (2007) Lumbar Vertebral Hemangioma Presenting with the Acute Onset of Neurological Symptoms. Journal of Neurosurgery: 7:80-85

2. Cheung N.K., Doorenbosch X.and Christie J.G.,2011.Rapid onset aggressive vertebral haemangioma. 27: 469-72

3. Ross J.S., Masaryk T.J., Modic M.T., Carter J.R., Mapstone T. and Dengel F.H.,1987.Vertebral hemangiomas:MRimaging. Radiology

4. Laredo J.D., Reizine D., Bard M. and Merland J.J.,1986.Vertebral hemangioma: radiologic evaluation. Radiology, 161:183189.

5. Tomita, K., Kawahara, N., Baba, H., Tsuchiya, H., Fujita, T. and Toribatake, Y. (1997) Total En Bloc Spondylectomy:A New Surgical Technique for Primary Malignant Vertebral Tumors.

6. Schwartz TH, Hibshoosh H, Riedel CJ: Estrogen and progesterone receptornegative T11 vertebral hemangioma presenting as a postpartum compression fracture: case report and management. Neurosurgery 2000, 46:218-221 